\title{
Analysis of Temperature Dependence of Dynamic Loss Tangent ( $\tan$ ठ) Relating to Molecular Motion of Polymer Chain in Amorphous Region in Fiber and Fiber-forming Polymeric Materials
}

\author{
Part 1: Viscoelastic Body Composed of Single Relaxation Time \\ By Sei-ichi Manabe, Kenji Kamide and Chozo Nakayama, Member, TMSJ \\ Textile Research Laboratory, Asahi Chemical Industry Company Ltd., Hacchonawate 11-7, Takatsuki-shi, Osaka
}

Based on the Jounal of the Textile Machinery Society of Japan, Transactions, Vol. 30, No 3, T45-T54 (1977-3)

\begin{abstract}
A theoretical equation is derived to determine the $\tan \delta$-temperature (T) curve of the system composed of a single relaxation time, and the curve is calculated. Comparison of the calculation with experimental results shows that: (1) The three-element model combining the Maxwell element with one spring parallelly is most suitable for a viscoelastic model having a peak in the $\tan \delta$-T curve. (2) The peak temperature in the $\tan \delta$-T curve, $T_{\max }(k)$, and the apparent activation energy $\Delta H a$, give the inherent half value width of $\tan \delta$-T curve $\Delta T_{1 / 2(s)}$ by the equation $\Delta T_{1 / 2(s)}=5.24 \times 10^{-3} T_{\max }^{2} / \Delta H a$. (3) The $\log \tan \delta$-T curve can be approximated by an equilateral triangle. (4) The parameter $B_{\infty}$, defined by the equation $B_{\infty}=522 \Delta \mathrm{Ha} / T_{\mathrm{max}}$, is convenient for determining the mechanism of viscoelastic absorption. (5) The average $\Delta T_{1 / 2(s)}$ of various polymers is $13 \mathrm{deg}$ for the absorption $\left(\alpha_{a}\right)$ related to the microbrownian movement of segment in amorphous, and $20 \mathrm{deg}$ for the absorption $\left(\beta_{a}\right)$ related to the local movement of the main chain. Thus, the value of $\beta_{a}$ absorption is larger than that of $\alpha_{a}$ absorption. (6) A method is presented to determine the relaxation time at the glass transition temperature $T_{g}$ by $\Delta H a$ and $T_{\max }$.
\end{abstract}

\section{Introduction}

For the purpose of establishing the correlationship between the fine structure and the physical properties of polymers, it is of great importance to evaluate quantitatively the aggregated state of the molecular chains in the amorphous region of semi-crystalline polymer solids, especially of fibers and fiber-forming polymeric materials. In this series of the studies, we will deal with the fine structure of the amorphous region whose size is $10-1000 \AA$ order of magnitude.

Studies on the fine structure of fibers reported so far have been mainly limited to the crystal region. However, the regions which govern the processability of fibers are not only the crystal region but also the amorphous region. For instance, the amorphous region greatly contributes to the dyability and the hygroscopicity of fibers. The change of the breaking elongation of unoriented fibers in the simultaneous draft/false-twist processing due to the lapse of time and the shrinkage phenomenon of polyamide fibers in the manufacturing process, are closely related to their amorphous regions.

The methods for determining the fine structure in the crystal region (e.g., crystallinity, crystal size, crystal defects, conformation of molecular chain) have already been established satisfactorily. On the other hand, no method has not yet been fully established, for analyzying the fine structure in the amorphous region, although some tentative methods are proposed such as hygroscopicity (e.g., isothermal absorption curve $\left.{ }^{[1-4]}\right)$, iodo-absorbability or phenol-absorbability (e.g., isothermal absorption curve $\mathrm{e}^{[5,6]}$ ), dyability (e.g., diffusion rate of dyestuff, equilibrium degree of exhaustion of dyestuff ${ }^{[7-9]}$ ) and reactivity towards chemical agents (e.g., change in the amount of reacted-agent with time or its concentration $\left.{ }^{[10]}\right)$. These methods are principally based on the difference in the chemical reactivity of both regions which relates only indirectly to the fine structure of fibers. Therefore, we can not compare between the fine structures of polymer solids differing their chemical compositions by these methods. Also, undesirable change in the fine structure of samples during the measurement is unavoidable.

In contrast to this, the dynamic loss tangent $(\tan \delta)$ versus temperature $(T)$ curve is expected to reflect sensively the fine structure of the region, in which the dynamic absorption denoted by $\alpha_{a}$ absorption is originated by the microbrownian motion of polymer chains in the amorphous region. Fukuda \& Ohmori ${ }^{[11]}$ and Kamide, et al. ${ }^{[12]}$, for example, demonstrated experimentally that the degree of exhaustion of disperse dyes is proportional to the integrated value of $\tan \delta$ over the temperature region from $T_{2}$ to $T_{1} ; \int_{T_{2}}^{T_{2}} \tan \delta d T$. 
Here $T_{1}$ is defined as the lower limit of $\alpha_{a}$ absorption. $T_{2}$ is decided experimentally, and depends on the molecular size of dyestuff and the exhaustion time and temperature. However, it should be pointed out that the physical meaning of the quantity $\int_{T_{1}}^{T_{2}} \tan \delta d T$ is not clear, and no theoretical background has not yet been given for integrating $\tan \delta$ with temperature. The lack of the fundamental theory on the tan $\delta$ - $T$ curve is mainly due to the following reasons:

(1) As is clear from the theory of linear viscoelasticity, $\tan \delta$ does not play an important role in the viscoelastic functions of the system in a strict sense, because $\tan \delta$ is only a parameter representing the loss component in the complex modulus and can not be reduced to any other fundamental function such as relaxation spectrum $H(\ln \tau)$. Therefore, when we put strain as excitation and stress as response, it is theoretically recommended to use storage modulus and loss modulus (E") for describing the viscoelastic properties.

(2) When the system is composed of many components with different relaxation times, we can not calculate theoretically the $\tan \delta$ value of the whole system, even if we know each $\tan \delta$. In contrast to this, other fundamental functions (e.g., $\left.E^{\prime}, E ”, H(\ln \tau)\right)$ are able to be calculated easily under the suitable assumptions such as the stress additivity or strain additivity from the corresponding values of components.

As $\tan \delta$ is principally independent of the sectional area of the sample, it can be more accurately measured than $E$ ' and $E$ " as far as the commercially available measuring aparatus of viscoelasticity concerns. So, $\tan \delta$ is widely adopted in order to deduce qualitatively the heterogeniety of amorphous regions in fibrous or irregularly-shaped samples despite of its ambiguity of physical meaning.

This series of papers intends to show both the basic theoretical equation for the $\tan \delta$ - $T$ curve and the result of numerical calculation by this equation and also to present a new method for evaluating the fine structure in amorphous regions from the observed $\tan \delta-T$ curve.

In this paper, the theoretical equation and subsequent numerical calculation by it will be given for the viscoelastic body having only one relaxation time.

\section{Theory}

In Fig. 1 all the possible three-element models with a single relaxation time $\tau$ are shown. $E_{1 a}$ and $E_{2 a}$ are elastic moduli of springs, $\eta_{2 a}$ the viscosity of the dashpot, in model (a). Hereafter, suffixes $b, c$, and $d$ denote models (b), (c), and (d), respectively. So, when the following equations hold, models (a) and (b) are completely equivalent to each other:

$$
\begin{aligned}
& E_{1 b}=E_{1 a}+E_{2 a} \\
& E_{2 b}=E_{1 a}\left(E_{1 a}+E_{2 a}\right) / E_{2 a} \\
& \eta_{2 b}=\left\{\left(E_{1 a}+E_{2 a}\right) / E_{2 a}\right\}^{2} \eta_{2 a}
\end{aligned}
$$

Model (c) is also equivalent to model (d) if $E_{1 c}, E_{2 c}$ and $\eta_{2 c}$
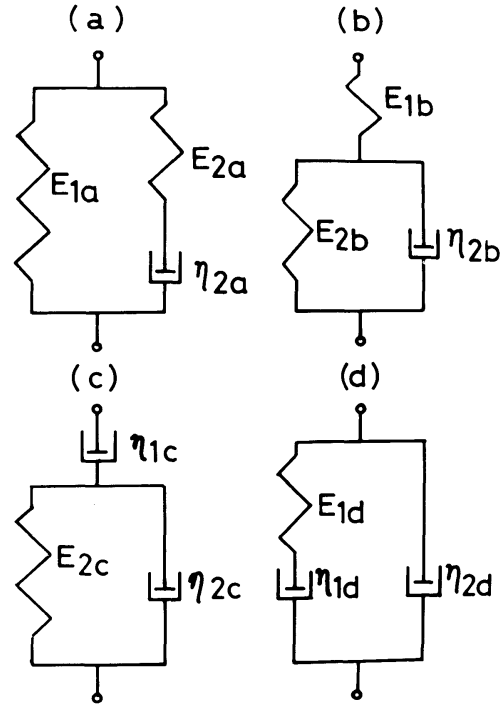

(d)

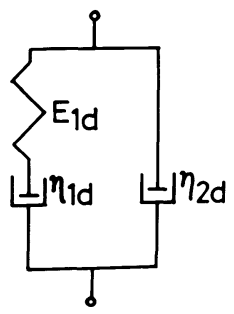

Fig. 1 Three element models with a single relaxation time: $E$; elastic modulus of spring, $\eta$; viscosity coefficient of dashpot

are given by equations analogous to (1)-(3) (not shown here). Consequently, models (a) and (c) are regarded as the basic model among any three element model with a single relaxation time. After simple calculation we know that the $\tan \delta$ - $T$ curve for model (c) has no maximum (peak) but a minimum. Since the system is composed of such elements as having only one relaxation time, $\tau_{1 c}$ should be proportional to $\eta_{2 c}$.

For a polymer solid many kinds of dynamic absorption have been observed in the $\tan \delta-T$ curve. For simplicity, we will discuss here the case in which three mechanical absorptions, $\alpha_{1}, \alpha_{2}$, and $\alpha_{3}$ from higher temperature side, are observed in this curve and their temperature locations are apart sufficiently from each other. One Maxwell element may be assigned to each of these three absorptions, and three elements thus assigned are assumed to be connected in parallel like the generalized Maxwell model. $\tau_{1}, \tau_{2}$, and $\tau_{3}$ are denoted as relaxation times of $\alpha_{1}, \alpha_{2}$, and $\alpha_{3}$ absorptions, respectively. When the measured temperature is near $T_{\max }$ of $\alpha_{2}$ absorption, $\omega \tau_{1} \gg 1$ and $\omega \tau_{3} \ll 1$ hold. This condition deduces that the Maxwell element representing $\alpha_{1}$ absorption can be approximated by one spring, and the element of $\alpha_{3}$ absorption can be ignored because the viscosity of dashpot of this element becomes nearly zero: The viscoelastic properties of the sample can be expressed by model (a) in Fig. 1.

We adopt model (a) in this study for the following four reasons: (1) This model is suitable for describing the viscoelastic properties of the polymer solid having many mechanical absorptions as mentioned above. (2) It is composed of the minimum mechanical elements which give a peak on 
the $\tan \delta$ - $T$ curve. (3) It is easily developed to the generalized Maxwell model when strain is excitation. (4) We can estimate three parameters $\left(E_{1 a}, E_{2 a}\right.$, and $\left.\eta_{2 a}\right)$ by flash inspection of the experimental $E^{\prime}-T$ curve.

$E_{1 a}$ corresponds to the elastic modulus of the sample after relaxation of specific absorption in question, and is not always consistent with the equilibrium elastic modulus $E_{e}$ as generally observed in a cross-linked polymer. $E_{2 a}$ equals to the relaxation intensity, and $\eta_{2 a} / E_{2 a}$ is approximated to the relaxation time.

With regard to model (a), the calculation of $\tan \delta$ will be done by setting $E_{1 a}=E_{1}, E_{2 a}=E_{2}, \eta_{2 a}=\eta_{2}$ for simplicity, and

$$
\tan \delta=a_{s} \chi /\left\{\left(1+a_{s}\right) \chi^{2}+1\right\}
$$

where $\omega$ is measured angular frequency, $\tau=\eta_{2} / E_{2}, \chi=\omega \tau$, and elastic modulus ratio $a_{s}=E_{2} / E_{1}$. As shown in Appendix 1, eq. (5) holds:

$$
\left.\begin{array}{l}
(\tan \delta)_{\max }=a_{s}\left(1+a_{s}\right)^{-1 / 2} / 2 \\
\Delta T_{1 / 2(s)}=5.24 \times 10^{-3} T_{\max } 2 / \Delta H a
\end{array}\right\}
$$

where suffix $s$ denotes the value of the system composing of a single relaxation time, and $\Delta H a$ means apparent activation energy ( $\mathrm{Kcal} / \mathrm{mol})$. In deriving eq. (5), temperature dependence of $\tau$ is assumed to be expressed by either Arrhenius (eq. (6)) or WLF type equation (eq. (7) $)^{[16]}$ :

$$
\begin{aligned}
& \tau=\tau_{\infty} \exp (\Delta H a / R T) \\
& \log \tau=\log \tau_{g}-C_{1}{ }^{g}\left(T-T_{g}\right) /\left\{C_{2} g+\left(T-T_{g}\right)\right\}
\end{aligned}
$$

where $\tau_{\infty}$ and $\tau_{g}$ are relaxation times at $T=\infty$ and $T=T_{g}$, respectively. $T_{g}$ is glass transition temperature, $C_{1^{g}}, C_{2^{g}}$ are constants appearing WLF equation, and $C_{1}{ }^{g}=17$ and $C_{2}{ }^{g}=51.6$ are employed in practical calculation. Hereafter we attempt to calculate the $\tan \delta$ - $T$ curve in two cases; Arrhenius and WLF type.

2.1 Case when the temperature-dependence of $\tau$ obeys Arrhenius type equation.

Eq. (8) is derived by putting $\tau$ of eq. (6) into eqs. (4) and (5):

$$
\tan \delta=2(\tan \delta)_{\max } \frac{\exp \left\{(\Delta H a / R)\left(1 / T-1 / T_{\max }\right)\right\}}{1+\exp \left\{(2 \Delta H a / R)\left(1 / T-1 / T_{\max }\right)\right\}}
$$

The influence of $\omega$ on shape and temperature location of the $\tan \delta$-T curve is equivalent to that of $T_{\max }$ on them. $T_{\max }$ is given by eq. (9) which is derived from eq. (8) as shown in Appendix 2:

$$
T_{\max }=-(\Delta H a / R) /\left\{\ln \left(1+a_{s}\right)^{1 / 2}+\ln \omega \tau_{\infty}\right\}
$$

Equation (9) indicates that $T_{\max }$ is proportional to $\Delta H a$ when $\tau_{\infty}, \omega$, and $a_{s}$ are kept constant, since the value of the denominator is alway negative. $\tau_{\infty}$ and $a_{s}$ are regarded as the function dependent only on absorption mechanism and absorption intensity, respectively. From experimental results it is evident that the relation of $\left|\ln \left(1+a_{s}\right)^{1 / 2}\right| \ll \mid \ln$ $\omega \tau_{\infty} \mid$ holds, and therefore, $T_{\max }$ values of $\tan \delta$-T curves concerning to the identical absorption mechanism for various polymer species should be proportional to $\Delta H a$ as far as the viscoelasticity of the sample can be described by the Maxwell element with a single relaxation time and eq. (6) holds.

2.2 Case when the temperature dependence of $\tau$ obeys WLF type equation

By combining eqs. (4), (5) and (7), we obtain

$$
\begin{aligned}
& \tan \delta=2(\tan \delta)_{\max } \\
& \quad \frac{\exp \left\{\left(\Delta H a^{w} \cdot \Delta H a, \mathrm{~m}^{w}\right)^{1 / 2}\left(1 / T_{\max }-1 / T\right) / R\right\}}{1+\exp 2\left\{\left(\Delta H a^{w} \cdot \Delta H a, m^{w}\right)^{1 / 2}\left(1 / T_{\max }-1 / T\right) / R\right\}}
\end{aligned}
$$

where $\Delta H a^{w}$ means the apparent activation energy calculated by the equation derived from WLF equation (see eq. (A. 1-14), and $\Delta H a, m^{w}$ is $\Delta H a^{w}$ at $T=T_{\max }$.

In order to simplify the numerical calculation, we employ eq. (4) and eq. (11) in place of eq. (10), since eq. (10) include $\Delta H a^{w}$ which depends on temperature in the complicated manner as shown in eq. (A. 1-13).

$$
\log \chi=\log \omega \tau_{g}-C_{1}{ }^{g}\left(T-T_{g}\right) /\left\{C_{2}^{g}+\left(T-T_{g}\right)\right\}
$$

Eq. (12) is derived from eq. (9) according to the procedure in Appendix 2.

$$
\begin{aligned}
& T_{\max }=-\left(\frac{C_{2}^{g} \cdot \Delta H a}{2.303 R C_{1}{ }^{g}}\right)^{1 / 2} \\
& \quad\left\{\ln \left(1+a_{s}\right)^{1 / 2}+\ln \omega \tau_{g}-2.303 C_{1}{ }^{g}\right\}
\end{aligned}
$$

$T_{\max }$ is proportional to $\Delta H a^{1 / 2}$ under the conditions that $a_{s}, \omega$, and $\tau_{g}$ are kept constant.

Comparison of the linearity of the plots of $T_{\max }$ vs. $\Delta H a^{1 / 2}$ (c.f., eq. (12)) with that of $T_{\max }$ vs. $\Delta H a$ (c. f. eq. (9)) enables us to determine which type, Arrhenius or WLF, is preferable in order to express the temperature dependence of relaxation time.

\section{Results of Numerical Calculation}

\subsection{Calculated $\tan \delta-\omega \tau$ curve}

In Fig. 2 is shown $\tan \delta-\omega \tau(=\chi)$ curves calculated by eq. (4) for a constant $a_{s}$. Numbers in the figure indicate the $a_{s}$ value. With the increase in $a_{s},(\tan \delta)_{\max }$ increases as is expected from eq. (5). It has been widely believed from ana$\operatorname{logy}$ to $E$ " $-T$ curve that the $\tan \delta$ peak is located at $\chi(=$ $\left.\chi_{\max }\right)=1$, but this is not true in the strict sense. The value of $\chi_{\max }$, corresponding to $(\tan \delta)_{\max }$, decreases with the increase of $a_{s}$ as is shown by

$$
(\tan \delta)_{\max }=\left(1-\chi_{\max }^{2}\right) / \chi_{\max } .
$$

Equation (13) is derived from eqs. (5) and (A. 1-1), and indicates $\chi_{\max } \leqq 1$, that is, $\omega \tau_{m} \leqq 1$.

The $\tan \delta$ value in Fig. 2 approaches to zero at larger value of $\chi$. When $a_{s} \geqq 3.2$, the $\tan \delta$-T curve is fairly symmetrical with respect to the peak. But when $a_{s}$ decreases, this symmetry becomes poor. The $\tan \delta$ versus frequency $(\omega)$ curve is analogous to that in Fig. 2. at a fixed temperature, since 
$\tau$ is constant at constant temperature. So, the abscissa $(\omega \tau$ axis) of Fig. 2 is changeable to the frequency axis by multiplying a factor of $\tau$. Naturally, the $\tan \delta$ - $\omega$ curve is independent of $\Delta H a$ as seen in eq. (4).

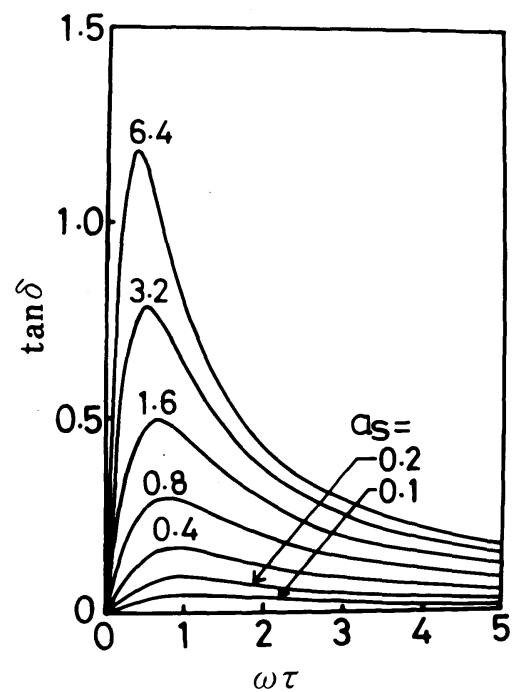

Fig. 2 Theoretical $\tan \delta$ - $\omega \tau$ curves of the model (a) in Fig. 1

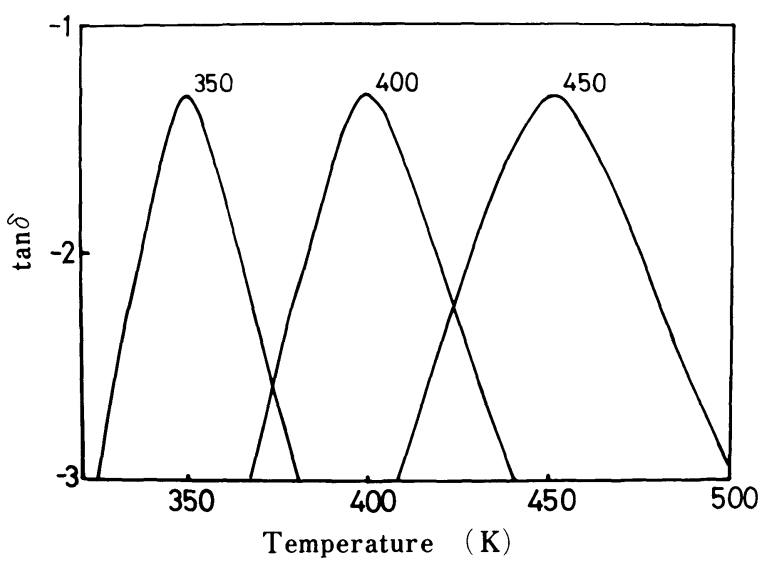

Fig. 3 Theoretical $\tan \delta$-T curve calculated by eq. (8): Numbers denote $T_{\max }$ in $K . a_{s}=0.1, \Delta H a=40 \mathrm{kcal} / \mathrm{mol}$

3.2 Calculated $\tan \delta$ - $T$ curve when the temperature dependence of $\tau$ obeys the Arrehnius

Some examples of $\tan \delta$-T curves calculated by eq. (8) are demonstrated in Fig. 3 when $(\tan \delta)_{\max }=0.0477$ (i.e., $a_{s}=0.1$, see eq. (5)), $\Delta H a=40 \mathrm{Kcal} / \mathrm{mol}$. Numbers in this figure denote $T_{\max }, 350,400$, and $450 \mathrm{~K}$. By eq. (5), $a_{s}$ can be determined from the observed $(\tan \delta)_{\max }$ value. Since /n $\left(1+a_{s}\right)^{1 / 2} /$ is negligibly small compared with $/ \ln \omega \tau_{\infty} /$ and $\omega$ is obtainable experimentally, $\ln \tau_{\infty}$ can be determined from experimental value of $\Delta H a / T_{\max }$ by eq. (9).
The higher the $T_{\max }$ of $\log \tan \delta$ - $T$ curve, the broader its shape is as is illustrated in Fig. 3. The curve can be reasonably approximated to a triangle. Here, we define $T_{l}$ and $T_{h}\left(T_{l}<T_{h}\right)$ as the temperatures at which the relation of $\log$ $\tan \delta=\log (\tan \delta)_{\max }-1$ holds. Then, $\Delta T_{l}=T_{\max }-T_{l}$ and $\Delta T_{h}=T_{h}-T_{\max }$ are calculated. In Fig. $4, T_{\max }$ and $\Delta H a$ dependence on $\Delta T_{l}$ (full line) and $\Delta T_{h}$ (broken line) are graphed. As $\Delta T_{l} \fallingdotseq \Delta T_{h}$ holds, the log $\tan \delta$ - $T$ curve can be represented by an equilateral triangle with high accuracy.

Fig. 5 shows the $\log \tan \delta$ - $T$ curve calculated for various $\triangle H a$ values in the region from 20 to $150 \mathrm{Kcal} / \mathrm{mol}$. The increase of $\triangle H a$ narrows the shape of the curve.

Fig. 6 shows the dependence of the $\tan \delta$-T curve on $a_{s}$, $\left(\Delta H a=50 \mathrm{Kcal} / \mathrm{mol}, \ln \tau_{\infty}=-46.06 \mathrm{sec}\right)$. The increase in

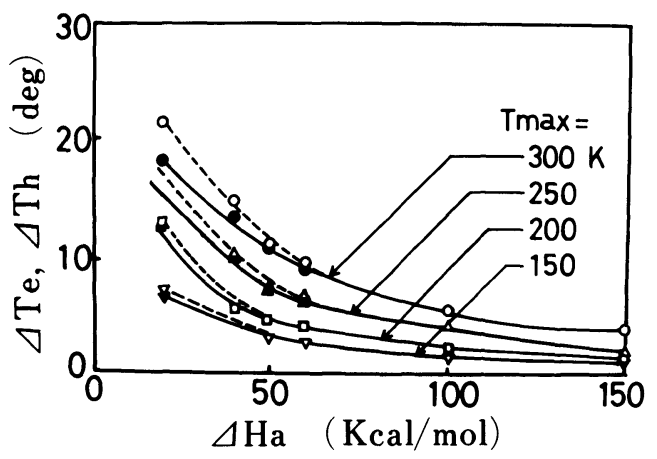

Fig. $4 \Delta H a$ dependence of $\Delta T_{1}$ and $\Delta T_{h}$ : Full line; $\Delta T_{l}$, broken line; $\Delta T_{h}$

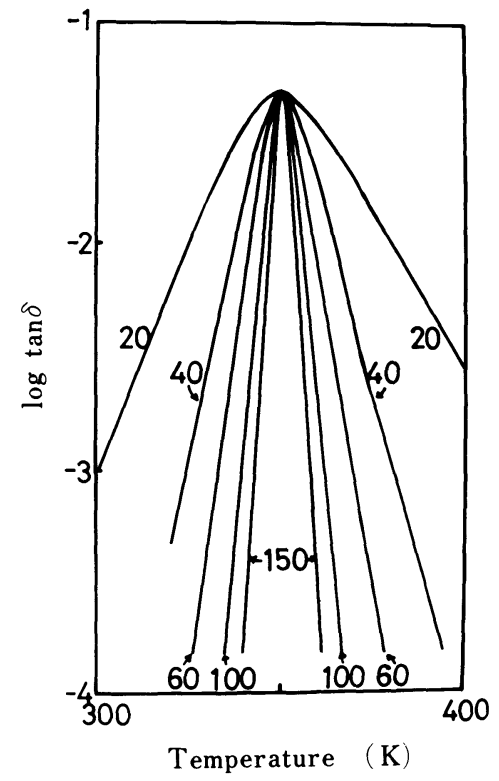

Fig. 5 Change of theoretical $\tan \delta$-T curves with $\Delta H a$ : Numbers in the figure denote values of $\Delta H a$ in $\mathrm{Kcal} / \mathrm{mol} . a_{\mathrm{s}}=0.1$, $T_{\max }=350 \mathrm{~K}$ 


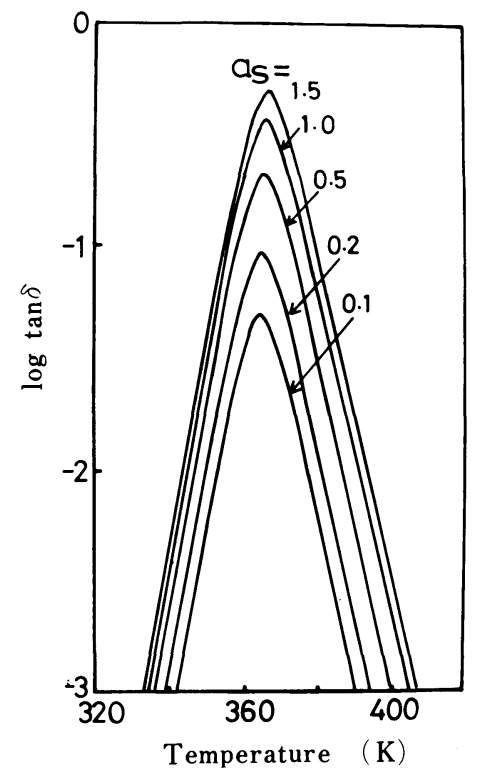

Fig. $6 a_{s}$ dependence of theoretical $\tan \delta$-T curve: $\Delta H a$ is $50 \mathrm{Kcal} /$ mol, and $\ln \tau_{\infty}$ is $-46.06 \mathrm{sec}$.

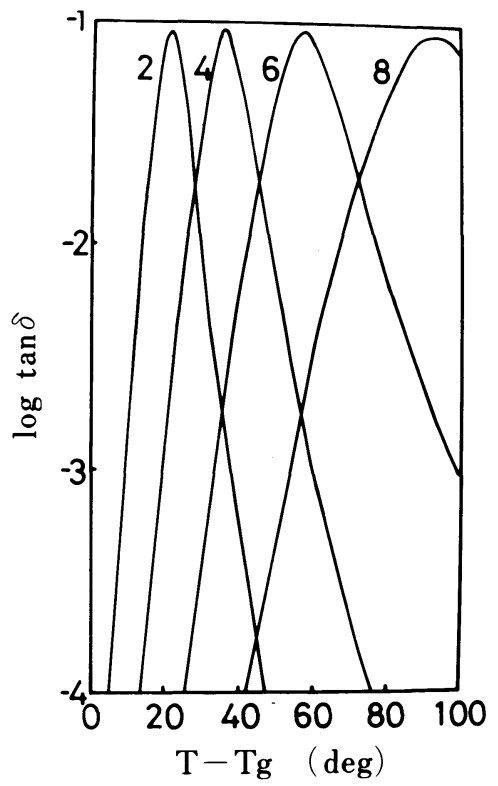

Fig. 7 Theoretical $\tan \delta$ - $\left(T-T_{g}\right)$ curve: Numbers in this figure indicate values of $\log \tau_{g}$ in sec. $a_{s}$ is $\mathbf{0 . 2}$

$a_{s}$ makes $(\tan \delta)_{\max }$ large monotonously resulting in the slight shift of $T_{\max }$ to the higher temperature side. This kind of $T_{\max }$ shift is of the order of only $3 \mathrm{deg}$., even if $a_{s}$ changes from 0.1 to 1.5 .

3.3 Calculated $\tan \delta-T$ curve when the temperature dependence of $\tau$ obeys the WLF

As is obvious from eq. (11), $\tan \delta$ is a function of $a_{s}, \omega$, $\tau_{g}$, and $\left(T-T_{g}\right)$. Therefore it is recommended to use the $\tan$ $\delta$ - $\left(T-T_{g}\right)$ curve instead of the $\tan \delta-T$ curve in order to determine the quantitative relationships between $\tan \delta$ and the characteristic values such as $a_{s}, \omega$, and $\tau_{g}$ of a viscoelastic body. Here, we will discuss only the case where $\omega$ is constant (690.8 rad/sec). It should be stressed that the limitation of this constancy is not important, since the $\tan \delta-T$ curve at other frequency can be easily calculated from the $\tan \delta-T$ curve at $\omega=690.8 \mathrm{rad} / \mathrm{sec}$ by using eq. (11), if it is needed. Change in $\omega$ corresponds to the change in $\tau_{g}$ from the point of view of numerical calculation of $\tan \delta$ - $T$ curves.

In Fig. $7, \log \tan \delta-\left(T-T_{g}\right)$ curves are shown in the $\log \tau_{g}$ range from 2 to $8(\mathrm{sec})$ at fixed $a_{s}(0.2)$. The curve can be approximated to a triangle. Therefore in the case where temperature dependence of $\tau$ is expressed by either Arrhenius or WLF type, the $\log \tan \delta-T$ curve can be approximated by a triangle of $\Delta T_{h} / \Delta T_{l}=1.3$.

In the case where $\tau$ obeys the WLF type, the $\tan \delta-\left(T-T_{g}\right)$ curve is independent of $T_{g}$, although Arrhenius-type $\tan \delta-T$ curves with different $T_{\max }$ and constant values of $\omega, a_{s}$ and $\Delta H a$ are not superimposable with each other by shifting the curve along $T$ axis. With the increase of $a_{s}$, $(\tan \delta)_{\max }$ increases (see eq. (2)), yielding a slight shift of $T_{\max }$ to the higher temperature side similarly to the case of Arrhenius type.

In Fig. 8 is shown $\tau_{g}$ dependence of $\left(T_{\max }-T_{g}\right)$ at two $a_{s}$ values. The value of $\left(T_{\max }-T_{g}\right)$ is predominantly determined by $\tau_{g}$ value, almost independent of $a_{s}$. Relationships among these parameters are given in eq. (A. 2-3). Here, the value of $\left(T_{\max }-T_{g}\right)$ for $a_{s}=0$ is the extrapolated value because of no peak on the $\tan \delta$ - $T$ curve at $a_{s}=0$.

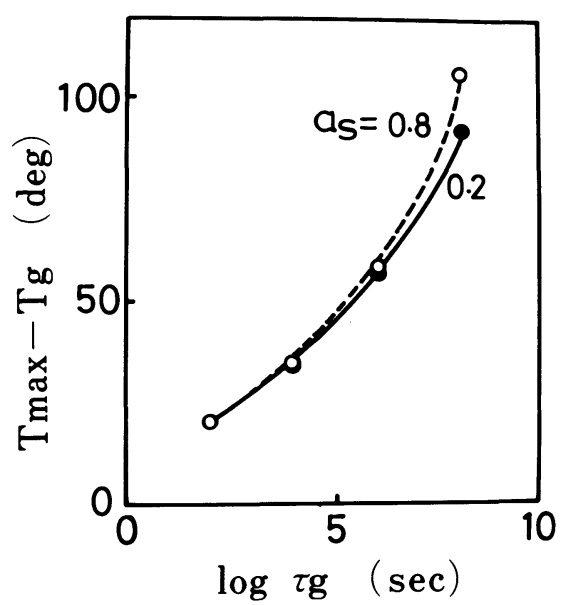

Fig. $8 \log \tau_{g}$ dependence of $\left(T_{\max }-T_{g}\right)$ : Measured frequency is $690.8 \mathrm{rad} / \mathrm{sec}$.

\section{Application to Experimental Data}

As far as the time-temperature transformation is applicable, temperature rise narrows the distribution of relaxation 
time, and consequently, the distribution function can be approximated by a $\delta$ function at the temperature near $T_{\max }$. Therefore, various relationships (eqs. (5), (9), and (12) etc.), derived theoretically for a single relaxation time, are also satisfied by the $\tan \delta-T$ curve of real polymer systems. For example, $T_{\max }$ of $\alpha_{a}$ absorption shifts to the higher temperature side with the increase of $\Delta H a$, and is nearly propotional to $\Delta H a$ (eq. (9)) or $\Delta H a^{1 / 2}$ (eq. (12)). Furthermore, the observed half value width of the $\tan \delta$-T curve of $\alpha_{a}$ is always larger than $\Delta T_{1 / 2(s)}$ obtained from eq. (5): the latter is the minimum $\Delta T_{1 / 2}$ and may be mamed as intrinsic half value width of the $\tan \delta$ - $T$ curve.

A few examples of the application of some relationships derived in section 2 to experimental data will be given below.

\subsection{Comparison between Arrhenius and WLF type}

In the above theory, we assumed that the temperature dependence of $\triangle H a$ was known in advance. Thus, when we apply any relationship to the experimental $\tan \delta$ - $T$ curves observed for real polymers, the temperature dependence of $\Delta H a$ for the system should be determined experimentally in any case. To do so, it is necessary to measure the temperature dependence of $\tau$ in the wide range of tempetature. In practice, $\Delta H a$ is calculated by,

$$
\Delta H a=R d \ln a_{T} / d(1 / T)
$$

where $a_{T}$ is a shift factor obtained by superimposing the $\tan \delta$ - $\omega$ curve at various fixed temperature. More conveniently, $\Delta H a$ can be estimated by putting the data of $\omega$ dependence of $T_{\max }$ into

$$
\Delta H a=R d \operatorname{lnf} / d\left(1 / T_{\max }\right) .
$$

Equation (14') is derived by replacing $T$ and $a_{T}$ in eq. (14) with $T_{\max }$ and $f$, respectively. When $\triangle H a$ is known, it is possible to judge by using the data of $T_{\max }$ and $\triangle \mathrm{Ha}$ values which type, Arrhenius or WLF, is more preferable for representing the temperature dependence of $\tau$.

By plotting experimental $T_{\max }$ values against $\Delta H a$ values obtained from literatures for amorphous and semi-crystalline polymers, we obtained the relation of $T_{\max }=450$ $\Delta H a^{1 / 2}$ for $\alpha_{a}$ absorption and $T_{\max }=77 \Delta H a$ for $\beta_{a}$ absorption. This $\beta_{a}$ is originated by local twisting vibration of polymer chains. The number of data were 33 for $\alpha_{a}$ and 22 for $\beta_{a}$ absorption.

Comparing these experimental relations of $T_{\max }$ and $\Delta H a$ with eqs. (9) and (12), it may be concluded that the temperature dependence of $\tau$ obeys the WLF for $\alpha_{a}$ absorption, and the Arrhenius type equation for $\beta_{a}$ absorption.

\subsection{Method for determining the absorption mechanism by}

the $\tan \delta$ - $T$ curve

The first step in the analysis of the $\tan \delta$ - $T$ curve is to study whether the $\tan \delta-T$ curve consists of only one absorption or not. Since $\log \tan \delta-1 / T$ and $\log \tan \delta-T$ curves can be approximated by equilateral triangles in case of a single re- laxation time, the asymmetry of these curves indicates the possibility of coexisting two or more absorptions. When the sample consists of a single relaxation time, its $\tan \delta$ - $T$ curve becomes narrower with decreasing $\omega$ as is easily speculated from the result in Fig. 3. So, it may be concluded that the $\tan \delta$ - $T$ curve consists of two or more absorptions, when the curve becomes broad with decreasing $\omega$.

The crystallinity dependence of the absorption intensity makes it clear that the given absorption is originated either by the motion of molecular chains in amorphous regions or by that in crystal regions. When the absorption is originated by the molecular motion in amorphous regions, let us propose a new method by which the absorption is distinguished between $\alpha_{a}$ and $\beta_{a}$. Here we define a new parameter $B_{\infty}$ which is given by $522 \Delta H a / T_{\max }$. The absorption in question can be classified into $\alpha_{a}$ or $\beta_{a}$ by this $B_{\infty}$. The coefficient of 522 is derived from the numerical calculation ${ }^{[14,15]}$ of the $\tan \delta$-T curve by Rouse theory, by which the relation of $B_{\infty}=$ $\ln a_{o}{ }^{2} \varepsilon_{\infty} / 6 \pi^{2} k=-\ln \tau_{\infty}-1$ holds. Here, $a_{o}$ is the end to end distance of the segment, $\varepsilon_{\infty}$ the viscosity coefficient of segmental motion at $T \rightarrow \infty$, and $k$ Boltzmann constant.

In Table 1 are listed the observed values of $\triangle H a$ and $T_{\max } / T_{m}$ ( $T_{m}$ is melting temperature) and calculated values of $B_{\infty}$ for $\alpha_{a}$ and $\beta_{a}$ absorptions. Since amorphous polymer does not show melting, the judgement of absorption mechanism by $T_{\max } / T_{m}$ is greatly restricted in practice.

Table 1 Experimental values of $\Delta H a, T_{\max } / T_{m}$, and calculated values of $B_{\infty}$ for $\alpha_{a}$ and $\beta_{a}$ absorptions

\begin{tabular}{llll} 
Absorption & $\Delta H a(\mathrm{Kcal} / \mathrm{mol})$ & $T_{\max } / T_{m}$ & $B_{\infty}(\mathrm{Kcal} / \mathrm{mol} . \mathrm{K})$ \\
\hline$\alpha_{a}$ & $0-150$ & $0.2-0.9$ & $15-200$ \\
$\beta_{a}$ & $0-35$ & $0.2-0.7$ & $0-65$ \\
\hline
\end{tabular}

Figs. 9(a) and 9(b) show the normalized frequency distribution curves of $\Delta H a$ and $B_{\infty}$ of $\alpha_{a}$ and $\beta_{a}$ absorptions, which are obtained by employing 33 data for $\alpha_{a}$ absorption and 22 data for $\beta_{a}$ absorption about polymer materials cited in literatures.

The probability to find from $\triangle H a$ value whether it belongs to $\alpha_{a}$ or $\beta_{a}$ is about $25 \%$. The corresponding probability to find it from $B_{\infty}$ is $33 \%$. So, the method by $B_{\infty}$ is more relaiable than the method by $\Delta H a$. From Fig. 9 we can conclude that (1) when $\triangle H a \geqq 35 \mathrm{Kcal} / \mathrm{mol}$, the absorption can be attributed to $\alpha_{a}$, (2) when $B_{\infty} \geqq 63$, the absorption is $\alpha_{a}$, and (3) when $B_{\infty} \leqq 31$, the absorption is $\beta_{a}$.

\subsection{Application to $\alpha_{a}$ and $\beta_{a}$ absorptions}

$T_{\max }$ shifts slightly to the higher temperature side with the increase of $(\tan \delta)_{\max }$ in both cases of Arrhenius and WLF type dependence of $\tau$. Accordingly, with the increase of the crystallinity $\chi_{c}$ (that corresponds to the decrease of $a_{s}$, it is expected theoretically that $(\tan \delta)_{\max }$ for $\alpha_{a}$ and $\beta_{a}$ ab- 


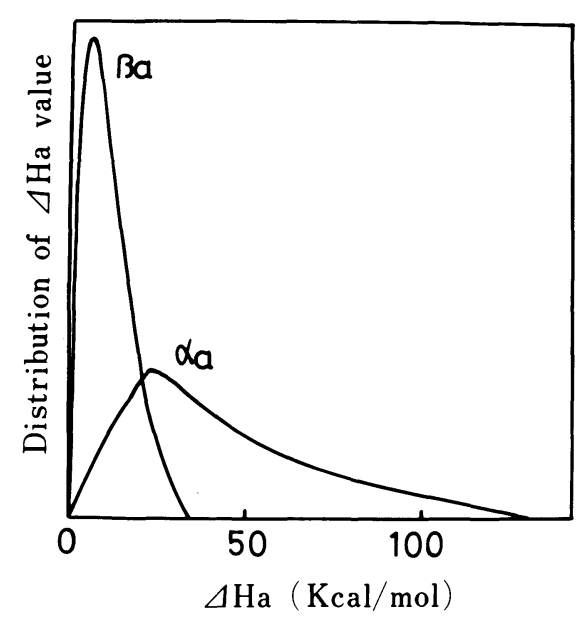

(a)

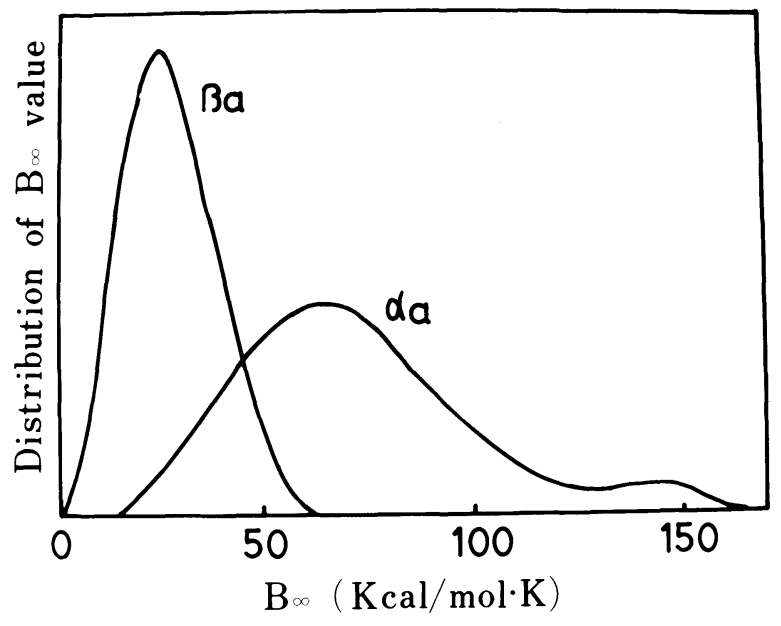

(b)

Fig. 9 Distribution curves of $\Delta H a$ and $B_{\infty}$ for $\alpha_{a}$ and $\beta_{a}$ absorptions observed for various polymers: sorptions decreases and $T_{\max }$ shifts to the lower temperature side.

Actually $(\tan \delta)_{\max }$ decreases with the increase in $\chi_{c}$ as is theoretically expected, but $T_{\max }$ shifts either to the higher temperature $\operatorname{side}^{[17]}$ or to the lower temperature side. ${ }^{[18]}$ The contradiction to the theory may be arised from the fine structural change in amorphous regions accormpanied by the change of $\chi_{c}$. The fine structural change may induce the changes in $\Delta H a$ and $\tau_{\infty}$ (or $\tau_{g}$ and $T_{g}$ ) at the same time.

The value of $\Delta T_{1 / 2(s)}$ was calculated by putting the experimental $\Delta H a$ and $T_{\max }$ data into eq. (5), and is the half value width inherent to the $\tan \delta$ - $T$ curve as mentioned above. When the experimental $\Delta T_{1 / 2}$ value is smaller than $\Delta T_{1 / 2(s)}$, the absorption may be induced by the resonance vibration in which the sample mass plays an important role.

In Table 2 are listed experimental $T_{\max }, \Delta H a, \Delta T_{1 / 2}$ for $\alpha_{a}$ and $\beta_{a}$ absorptions, and calculated $\Delta T_{1 / 2(s)} . \Delta T_{1 / 2(s)}$ for $\beta_{a}$ absorption (10-30 deg) is larger than that of $\alpha_{a}(5-20$ deg). The experimental tan $\delta$ - $T$ curves relating to $\beta_{a}$ absorption are known to be broad. This broadness has been interpreted to the due to the small size of the moving-unit of $\beta_{a}$ absorption ${ }^{[19]}$ : the motion of this unit is affected by the local heterogeniety in the fine structure and therefore gives rise to the broad distribution of relaxation time, resulting in the broad $\tan \delta-T$ curve for $\beta_{a}$ absorption. However, the large $\Delta T_{1 / 2(s)}$ value of $\beta_{a}$ absorption may be another reason for broadening the curve. This effect of broadening the inherent half value width is evident (20-30 deg) in the case of polystyrene and polymethylmethacrylate in Table 2 .

The values of $\left(T_{\max }-T_{g}\right)$ for $\alpha_{a}$ absorption obtained by putting experimental $T_{\max }$ and $\Delta H a$ into eq. (A. 1-14) are rather in the narrow range of $20-60 \mathrm{deg}$, and concentrated around 50 deg. Accordingly, $T_{\max }$ is higher than $T_{g}$ by about 50 deg.

When $\left(T_{\max }-T_{g}\right)$ is obtained by the above procedure, $\tau_{g}$ can be known from this value in Fig. 8. For example, log $\tau_{g} \fallingdotseq 5.3$ (sec) in the case of $T_{\max }-T_{g}=50 \mathrm{deg}$. The $\tau_{g}$ value has not yet been extensively measured so far. ${ }^{[20]}$ This is due to the complicated analytical procedure adopted. For

Table 2 Comparison of theoretical values of $\Delta T_{1 / 2(s)}$ with experimental values of $\Delta T_{1 / 2}$ in $\alpha_{a}$ and $\beta_{a}$ absorptions for various polymers $(110 \mathrm{~Hz})$

\begin{tabular}{lllcllllc}
\hline Polymer & \multicolumn{7}{c}{$\alpha_{a}$ absorption } & \multicolumn{7}{c}{$\beta_{a}$ absorption } \\
\hline & $T_{\max } *^{* 1}$ & $\Delta H a^{* 2}$ & $\Delta T_{1 / 2(s)} * 3$ & $\Delta T_{1 / 2} * 3$ & $T_{\max }$ & $\Delta H a$ & $\Delta T_{1 / 2(s)}$ & $\Delta T_{1 / 2}$ \\
\hline Polyethylene & 265 & 43 & 8.5 & $50-$ & 162 & 13 & 10.6 & $50-75$ \\
Polypropylene & 296 & 46 & 10.5 & $35-$ & 233 & 14 & 20.2 & $110-$ \\
Polyethyleneterephthalate*5 & 400 & 67 & 12.5 & $20-$ & 220 & 18 & 14.1 & 86.8 \\
Polyvinylchloride & 380 & 68 & 11.1 & $40-$ & 285 & 18 & 23.6 & $150-$ \\
Polymethylmethacrylate & 370 & 57 & 12.6 & 25 & 340 & 21 & 28.8 & $100-$ \\
Polystyrene & 404 & 129 & 6.8 & $14-$ & 350 & 30 & 21.4 & - \\
Polyvinylacetate & 330 & 40 & 14.3 & $45-$ & $-{ }^{* 4}$ & - & - & - \\
\hline
\end{tabular}

$*_{1}$ absolute temperature $(k),{ }^{* 2} \mathrm{Kcal} / \mathrm{mol},{ }^{* 3} \mathrm{deg},{ }^{* 4}$ unseparable from $\alpha_{a}$ absorption, ${ }^{* 5}$ crystallinity by $\mathrm{X}$-ray method is $0 \%$ 
example, it is in need to measure $T_{g}$ in addition to $E^{\prime}, E^{\prime \prime}-\omega$ curve or relaxation modulus-time curve, and then to estimate the $\chi$ value at temperature $T$ from above curves, and finally to calculate $\omega \tau_{g}$ by eq. (11). The method proposed here for estimating $\tau_{g}$ value has the merit to be able to decide it with simple analyzing procedure without knowing $T_{g}$ from $T_{\max }$ and $\Delta H a$.

\section{Conclusion}

Model (a) in Fig. 1 is most suitable to represent the $\tan \delta$ $T$ curve for the system in which more than two viscoelastic absorptions are observed. Theoretically, those $(\tan \delta)_{\max }$, $T_{\max }$, and $\Delta H a$ are the most important experimental values which characterize the $\tan \delta$ - $T$ curve. The experimental value of $\Delta T_{1 / 2}$ is larger than $\Delta T_{1 / 2(s)}$ because of a rough approximation that the distribution of relaxation time of the system can be represented by a single relaxation time. Therefore, quantitative discussion by considering that distribution is still left to open.

Appendix 1 Relationships between $a_{s}$ and $(\tan \delta)_{\max }, T_{\max }$, $\Delta T_{1 / 2(s)}$ in model (a) in Fig. 1

By applying to eq. (4) the relation of $d \tan \delta / d \chi=0$ at $\chi$ $=\chi_{\max }$, we obtain

$$
\chi_{\max }=\left(1+a_{s}\right)^{-1 / 2} .
$$

By substituting eq. (A. 1-1) into eq. (4), we derive

$(\tan \delta)_{\max }=a_{s} /\left\{2\left(1+a_{s}\right)^{1 / 2}\right\}$

The values of $\chi$ which satisfy the equation $(\tan \delta)_{\max }=$ $2 \tan \delta$ are given by

$$
a_{s} /\left\{4\left(1+a_{s}\right)^{1 / 2}\right\}=a_{s} \chi /\left\{\left(1+a_{s}\right) \chi^{2}+1\right\} \text {.......(A. 1-3) }
$$

Equation (A. 1-3) is derived by eqs. (4) and (A. 1-2). Two values of $\chi\left(\chi_{1}\right.$ and $\left.\chi_{2}\right)$ obtained from eq. (A. 1-3) are given

$$
\text { by } \chi_{1} / \chi_{\max }=(2-\sqrt{3}) / 2
$$$$
\chi_{2} / \chi_{\max }=(2+\sqrt{3}) / 2
$$

Thus, we have

$$
\ln \left(\chi_{2} / \chi_{1}\right)=\ln \{(2+\sqrt{3}) /(2-\sqrt{3})\}
$$

From eq. (6),

$$
\begin{aligned}
& \chi_{1}=\omega \tau_{\infty} \exp \left(\Delta H a / R T_{1}\right) \\
& \chi_{2}=\omega \tau_{\infty} \exp \left(\Delta H a / R T_{2}\right)
\end{aligned}
$$

Substituting eq. (A. 1-6) into eq. (A. 1-5), $T_{1}$ and $T_{2}$ can be expressed as

$$
\frac{1}{T_{2}}-\frac{1}{T_{1}}=\frac{R}{\Delta H a} \ln \frac{2+\sqrt{3}}{2-\sqrt{3}}
$$

when the approximation $T_{1} \cdot T_{2} \fallingdotseq T_{\max ^{2}}$ (A. 1-8) is adopted, eq. (5) holds. On the other hand, when $\tau$ is given by eq. (7), we have

$$
\begin{aligned}
& \log \chi_{1}=\log \omega \tau_{g}+C_{1}{ }^{g}\left(T_{1}-T_{g}\right) /\left\{C_{2}{ }^{g}+\left(T_{1}-T_{g}\right)\right\} \\
& \log \chi_{2}=\log \omega \tau_{g}+C_{1}{ }^{g}\left(T_{2}-T_{g}\right) /\left\{C_{2}{ }^{g}+\left(T_{2}-T_{g}\right)\right\}
\end{aligned}
$$

By substituting eq. (A. 1-9) into (A. 1-5), it goes.

$$
\frac{C_{1}{ }^{g}\left(T_{2}-T_{g}\right)}{C_{2}{ }^{g}+\left(T_{2}-T_{g}\right)}-\frac{C_{1}{ }^{g}\left(T_{1}-T_{g}\right)}{C_{2}{ }^{g}+\left(T_{1}-T_{g}\right)}=\log \frac{2+\sqrt{3}}{2-\sqrt{3}}
$$

In the case in which

$$
T_{2}+T_{1} \fallingdotseq 2 T_{\max },
$$

we have by substituting eq. (A. 1-8) into eq. (A. 1-11),

$$
\Delta T_{1 / 2(s)}=\frac{C_{2}{ }^{g}+T_{\max }-T_{g}}{C_{1}{ }^{g} \cdot C_{2}{ }^{g}} \log \frac{2+\sqrt{3}}{2-\sqrt{3}}
$$

Activation energy $\Delta \mathrm{Ha}^{w}$ is derived from eq. (7), and

$$
\Delta H a^{w}=2.303 R C_{1}^{g} \cdot C_{2}^{g} T^{2} /\left(C_{2}{ }^{g}+T-T_{g}\right)^{2} \text { (A. 1-13) }
$$

Assuming that $\triangle H a, m^{w}\left(\Delta H a^{w}\right.$ at $T=T_{\max }$ ) is equal to $\Delta H a$ in the Arrhenius type that is, when $\Delta H a, m^{w} \fallingdotseq \Delta H a$ (A. 1-14) holds, eq. (A. 1-7) is derived from eqs. (A. 1-13) and (A. 1-14).

\section{Appendix 2 Relationships between $\Delta H_{a}$ and $T_{\max }$}

When temperature dependence of $\tau$ obeys the Arrhenius type equation, we have from eq. (6),

$$
\chi_{\max }=\omega \tau_{\infty} \exp \left(\Delta H a / R T_{\max }\right)
$$

By putting eq. (A. 2-1) into eq. (A. 1-1), eq. (9) can be obtained. When the temperature dependence of $\tau$ obeys the WLF type equation, we have from eq. (7),

$$
\begin{aligned}
& \ln \chi_{\max }=\ln \omega \tau_{g}-2.303 C_{1}{ }^{g}\left(T_{\max }-T_{g}\right) /\left\{C_{2}{ }^{g}\right. \\
& \left.\quad+\left(T_{\max }-T_{g}\right)\right\}
\end{aligned}
$$

Equation (A. 2-3) is derived by substituting eq. (A. 2-2) into the left side term in eq. (A. 1-1).

$$
\begin{aligned}
& 1 / 2 \ln \left(1+a_{s}\right)+\ln \omega \tau_{g}=2.303 C_{1}{ }^{g}\left(T_{\max }-T_{g}\right) /\left(C_{2}{ }^{g}\right. \\
& \left.\quad+T_{\max }-T_{g}\right)
\end{aligned}
$$

We obtain eq. (12) by eliminating $T_{g}$ in eq. (A. 2-3) by eq. (A. 1-14).

\section{References}

[1] A. Koshima, T. Tagawa; J. Appl. Polymer Sci., 9, 45 and 117 (1965).

[2] H. W. Starkweather; J. Appl. Polymer Sci., 2, 129 (1959).

[3] R. Jeffries; J. Appl. Polymer Sci., 8, 1213 (1964).

[4] A. Takizawa, T. Negishi, K. Ishikawa; J. Polymer Sci., 6, A-1, 475 (1968).

[5] T. Haga; J. Soc. Fiber Sci. \& Tech. Japan, 31, T-86 (1975).

[6] K. Kitamura, S. Kobayashi, Z. Yoshida; J. Soc. Fiber Sci. \& Tech. Japan, 27, 501 (1971).

[7] S. Rosenbaum; J. Appl. Polymer Sci., 7, 1225 (1963).

[8] K. Kitamura; Dyeing Industry Japan, 17, 15 (1969).

[9] H. W. Peters, T. R. White; J. Soc. Dyers and Col., 77, 601 (1961).

[10] H. Maeda; J. Soc. Fiber Sci. \& Tech. Japan, 12., 6 (1956).

[11] T. Fukuda, S. Ohmori; J. Soc. Fiber Sci. \& Technol. 
Japan, 27, 83 (1971).

[12] K. Kamide, S. Manabe, T. Sakamoto; Preprint of conference in autumn of Soc. Fiber Sci. \& Tech. Japan, p. 50 (1971).

[13] S. Manabe, K. Kamide, C. Nakayama; J. Text. Mach. Soc. Japan, 30, T55 (1977).

[14] S. Manabe, K. Kamide, C. Nakayama; J. Text. Mach. Soc. Japan, 30, T66 (1977).

[15] S. Manabe, K. Kamide, C. Nakayama, S. Kobayashi; J. Text. Mach. Soc. Japan, 30, T85 (1977).
[16] J. D. Ferry; Viscoelastic Properties of High Polymers; 1962, New York, John Wiley \& Sons.

[17] C. D. Armeniades; J. Polymer Sci., A-2, 9, 1345 (1971).

[18] For example, Soc. Polymer Sci. Japan ed., Rheology Handbook, pp. 200 and 211, 1965, Maruzen.

[19] For example, Soc. Phys. Sci. Japan ed., Polymer Physics, p. 158, 1963, Asakura Syoten.

[20] A. V. Tobolsky, E. Cutsiff; J. Polymer Sci., 19, 111 (1965).

[21] P. E. Rouse; J. Chem. Phys., 21, 1272 (1953). 